\title{
Negative refraction in three-dimensional point-dipolelike polaritonic crystals
}

\author{
A. Rose ${ }^{1, a)}$ and K. Kempa ${ }^{2}$ \\ ${ }^{1}$ Boston College, Chestnut Hill, Massachusetts 02467, USA and Duke University, Durham, \\ North Carolina 27708, USA \\ ${ }^{2}$ Boston College, Chestnut Hill, Massachusetts 02467, USA
}

(Received 3 June 2010; accepted 10 September 2010; published online 1 November 2010)

\begin{abstract}
We propose two practical realizations of the point-dipole crystal, shown previously to be capable of negative refraction and other metamaterial and plasmonic properties. These are: an array of highly polarizable spheres, and the complementary array of spherical cavities in a highly polarizable medium. We study light propagation through these polaritonic crystals and show that they are capable of negative refraction, and can be constructed from available materials for experimental demonstrations at both microwave and optical frequencies. (C) 2010 American Institute of Physics.

[doi:10.1063/1.3500323]
\end{abstract}

\section{INTRODUCTION}

Negative refraction (NR), initially proposed by Veselago in the $1960 \mathrm{~s},{ }^{1}$ has recently attracted strong research interest. ${ }^{2-21}$ An exciting consequence of NR was discovered by Pendry, ${ }^{17}$ and involves the possibility of constructing a superlens capable of resolution surpassing the diffraction limit of conventional lenses. Various approaches have been taken to demonstrate NR. In one, an effective medium consisting of metallic split-ring resonators and wires was proposed. ${ }^{3-5}$ Similar ideas have been exploited in effective media made of spherical particles in a matrix..$^{6-9}$ In another approach, it was shown that NR occurs in photonic, ${ }^{19,21}$ and polaritonic crystals. ${ }^{22-24}$ Recently, Kempa et. al, ${ }^{25}$ investigated the electromagnetic response of a cubic point-dipole crystal, and showed that this is a polaritonic crystal that allows for bulk and surface plasmon wave propagation, in addition to the usual photonic crystal effects (bands, gaps, etc.). Its effective dielectric response is not only frequency, but also plasmon wave-vector dependent, and therefore this is a nonlocal polaritonic crystal. ${ }^{25}$ Furthermore, with the right choice of parameters, the point-dipole crystal is a metamedium, capable of NR. The key feature of this polaritonic crystal is that the light scattering is dominated by the intraparticle resonances, in contrast to the scattering in a photonic crystal, which is largely interparticle. As we show later, this important difference gives rise to a significant advantage, as there exist a variety of customizable nanoparticles with strong intraparticle resonances in the optical range. In addition, the point-dipole nature of the crystal allows for a simple theoretical analysis, which can be invaluable in the initial design of a desired medium (or metamedium). These benefits should increase both the feasibility and flexibility of an optical metamaterial based on our proposed crystals.

In this work, we propose to use polaritonic crystals ${ }^{26,27}$ to develop practical realizations of the dipolar crystal. The first is a three-dimensional lattice of small spheres made of a highly polarizable dielectric, and the second, a complemen-

${ }^{a)}$ Electronic mail: adr19@duke.edu. tary crystal of small spherical cavities in a highly polarizable medium. We simulate the response of such systems by employing the finite difference time domain, and the finite difference frequency domain computational schemes, ${ }^{22,28}$ and show that they support NR.

\section{THEORY}

The key to developing a dipole crystal analog based on highly polarizable materials is to exploit the dominant surface polarization in such materials. This polarization process, in the long-wavelength limit, can be visualized as a rigid shift of negative charges (electrons) against a background of positive charges. This leads to a significant build-up of charges at the surfaces, as illustrated in Fig. 1. The top section of Fig. 1(a) shows a schematic of an array of small spheres in the absence of an electric field. After the longwavelength electric field is applied, a rigid shift of electrons within the polarizable medium occurs, inducing dipolelike charge build-up on opposite surfaces of the spheres (colored figure available online). The corresponding set of images for (a)
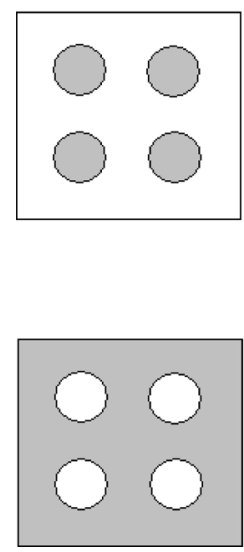

(b)
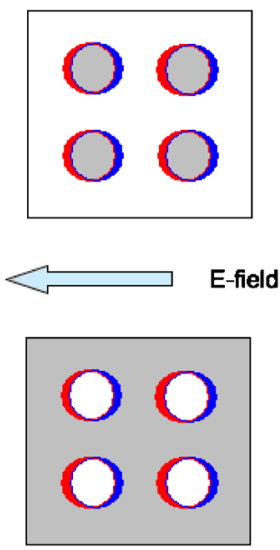

FIG. 1. (Color online) Schematic of the charge polarization in two complementary polaritonic crystals before (a), and after the electric field is applied (b). Top is the crystal of spheres, and bottom the crystal of cavities. 


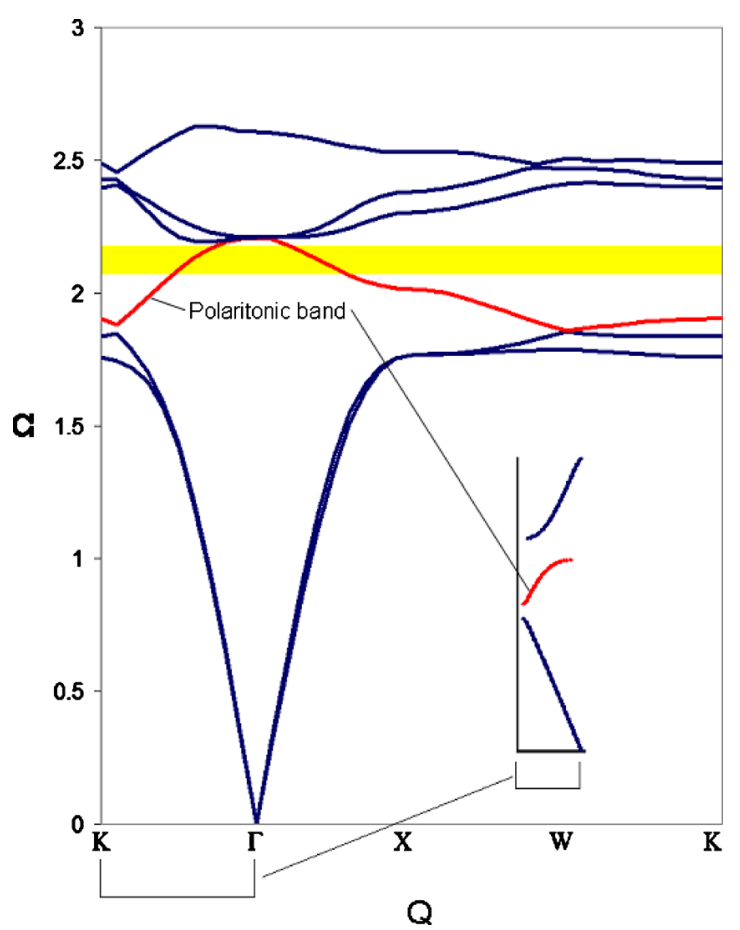

FIG. 2. (Color online) Polaritonic band structure of the fcc crystal of dielectric spheres. Parameters: $\varepsilon_{\mathrm{s}}=30, r=0.31 a$, and $\varepsilon_{\mathrm{b}}=1$. Inset: corresponding fragment of the band structure of a cubic crystal of perfect point-dipoles, obtained from Eq. (1). The dimensionless wave vector and frequency are $Q=k a$ and $\Omega=\omega a / c$, respectively.

an array of spherical cavities is shown in the bottom section of Fig. 1, demonstrating a similar process and end result. It follows that the two complementary crystals of spheres and cavities sketched in Fig. 1, made of highly polarizable dielectrics, can be expected to display similar characteristics to the point-dipole crystal of Ref. 25 .

Arrays of dielectric spheres have been shown to develop strong Mie resonances, ${ }^{29}$ and have been proposed as effective metamedia. ${ }^{24}$ Therefore, we chose as the first of the complementary point-dipolelike crystals the face centered cubic (fcc) lattice of highly polarizable spheres (dielectric constant $\varepsilon_{\mathrm{S}}=30$ ) of radius $r=0.31 a$ ( $a$ is the lattice constant), embedded in a dielectric matrix with $\varepsilon_{\mathrm{b}}=1$. It should be noted that because the curvature of the negatively dispersive band in a point-dipole crystal is controlled by intraparticle resonances, a strong Mie resonance is the most important characteristic of these spheres. For the proposed configuration, the first two Mie resonances correspond to $\Omega=1.80$ and $\Omega=2.52$, respectively, where $\Omega=\omega a / c$ is a dimensionless frequency, $\omega$ is the angular frequency of the radiation, and $c$ is the speed of light in vacuum. From Fig. 2, we can see that these frequencies correspond quite well to the band gaps along the $\Gamma-\mathrm{K}$ and $\Gamma-\mathrm{X}$ lines of symmetry. In addition, the symmetry of the charge oscillation in the bands is identical to that for the corresponding Mie resonances. For example, it has the dipolar character for the lowest resonance. Thus, as expected, the strong Mie resonances localize photons and cause "flattening" of the bands, which promotes the gap formation at the $\mathrm{K}$ point. Generally speaking, the polaritonic band is adjusted by tuning these resonances for a single sphere, while the unit-cell shape and dimensions control the periodicity-induced phase shift that cause this band to be negatively dispersive within the reduced Brillouin zone. This photonic crystal effect is in contrast to other Mie resonancebased metamaterials, which achieve NR by simultaneously exciting electric and magnetic resonances. As expected, the $\Gamma-\mathrm{K}$ portion of this plot is in good qualitative agreement with the corresponding dispersion of polaritonic modes in the strictly point-dipole, simple cubic crystal, shown in the inset of Fig. 2, and given by the fully retarded, analytical formula derived in Ref. 25

$$
Q=\arccos \left[\frac{B_{1} \pm \sqrt{B_{1}^{2}-8 v(1) B_{2}}}{4 v(1)}\right],
$$

where $Q=k a$ is the dimensionless form of the wave vector $k$. The remaining parameters are

$$
\begin{aligned}
& B_{1}=A+2 v(1) \cos \Omega, \\
& B_{2}=A \cos \Omega+\frac{1}{2} \Omega \sin \Omega, \\
& A=\frac{\Omega^{2}-\Omega_{0}^{2}}{\Omega_{p}^{2}}-v(0),
\end{aligned}
$$

with first two Fourier (cosine) amplitudes of the dipoledipole interaction given by $v(0)=-0.3594, v(1)=0.01303$, and $\Omega_{0}=\sqrt{\varepsilon_{b}} \omega_{0} a / c$, and $\Omega_{p}=\sqrt{\varepsilon_{b}} \omega_{p} a / c$. The dispersion branches shown in the inset in Fig. 2 are obtained by adjusting the resonance frequencies $\omega_{0}$ and $\omega_{p}$, as well as the background dielectric constant $\varepsilon_{b}$. Only qualitative agreement is expected between this calculated dispersion for a cubic crystal of perfect point-dipoles and the full simulations for the fcc crystal of spheres, but it allows identification of the negatively dispersing branch (denoted in Fig. 2) as the polaritonic band of interest for NR. ${ }^{25}$

\section{SIMULATION}

\section{A. Lattice of dielectric spheres}

We have verified that NR, with the effective refractive index $n=-1$, indeed occurs at the intersection of the vacuum light line with the negatively sloped, polaritonic band (i.e., at the frequency $\Omega=2.14$ ) for the chosen parameters of the system. However, the validity of this index of refraction requires some discussion. On the one hand, the proposed system is not perfectly isotropic. This effect is weakened due to the high symmetry of the fcc lattice, which allows for the construction of a nearly spherical equifrequency surface. ${ }^{19}$ In addition, the band structure in Fig. 2 demonstrates that the negative dispersive branch approaches isotropy in the limit

$Q \ll 2 \pi$.

This limit, in fact, is shared by the effective medium theory, which requires that the wavelength of radiation be much longer than the lattice constant for an effective set of parameters (i.e., refractive index) to be valid. In our system, the wavelength of the incident radiation is just a factor of 3 greater than the lattice constant. While this incomplete satisfaction of the limit will undoubtedly affect our results, the 


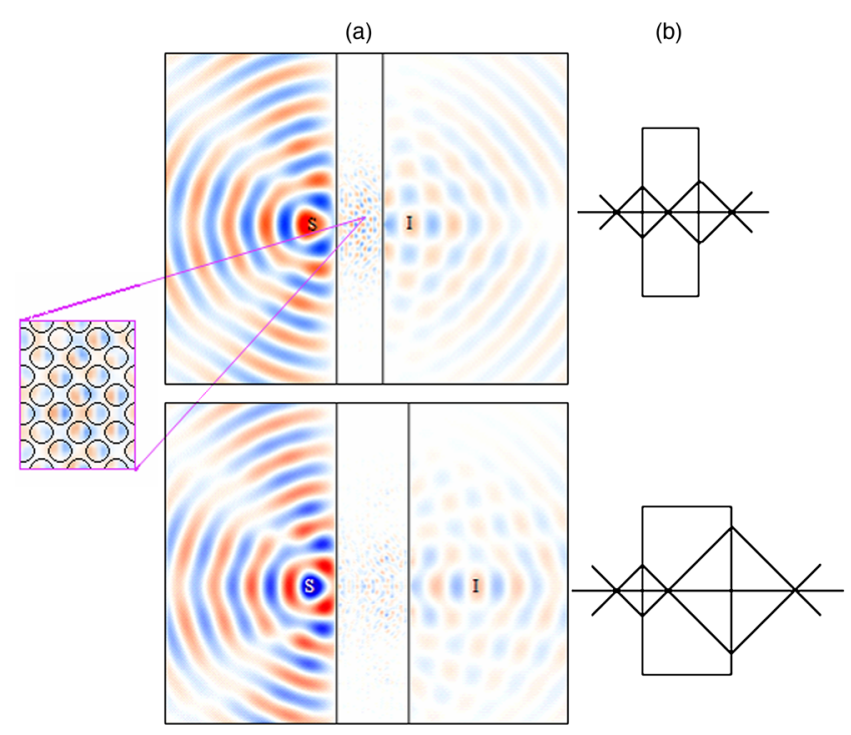

FIG. 3. (Color online) (a) Map of the z-component of the electric field of a wave propagating through the fcc crystal slab of spheres (parameters as in Fig. 2), at the frequency $\Omega=\omega a / c=2.14$, for two different thicknesses of the slab. Color intensity corresponds to electric field amplitude at an instant of time, while color change indicates reversal of the field component direction. The light source is located at $\mathrm{S}$, and the corresponding image at I. Inset shows a magnification of the slab, with sphere locations denoted by black circles. Colored figure available online. (b) Schematic of the light rays through corresponding slabs of a material with $n=-1$.

fact that this system's major constraints are lifted in the same limit presents a clear path to significant improvements through the use of composite particles, as will be discussed in Sec. IV. Figure 3(a) shows light propagation at this frequency through a thin slab of the crystal (thickness $w$ ). Shown is the map of the z-component of the electric field; color intensity corresponds to electric field amplitude at a specified instant of time, and the color change indicates reversal of the field component direction (colored figure available online). Light originates from a line source (extended along the $\mathrm{z}$ direction) located at the point $\mathrm{S}$, at a distance $d$ away from the surface of the slab. An image of the source forms to the right of the slab (marked I), indicating that NR is occurring within the crystal.

Furthermore, Fig. 3 confirms that this lensing is indeed unrestricted, i.e., it is not a result of the "channeling" effect. ${ }^{20}$ According to geometrical optics, for a material with an effective index of refraction $n=-1$, the image of a point source located to the left of the slab is expected to form on the opposite side of the crystal at a distance $x=w-d$ away from the right surface of the slab. Though the presence of strong Mie resonances precludes the use of geometrical optics for calculation of the fields within the crystal, it can still be used to predict the focal point outside of the slab. Figure 3(a) clearly demonstrates satisfaction of this requirement; the image positions, determined by the field maxima, correspond quantitatively to the calculated locations for two different slab thicknesses. Figure 3(b) shows sketches of the corresponding geometric optics constructions. We have also verified that the image formation follows the geometric optics rules when the distance between the source and the slab is varied. Though invariant to these changes, the image quality

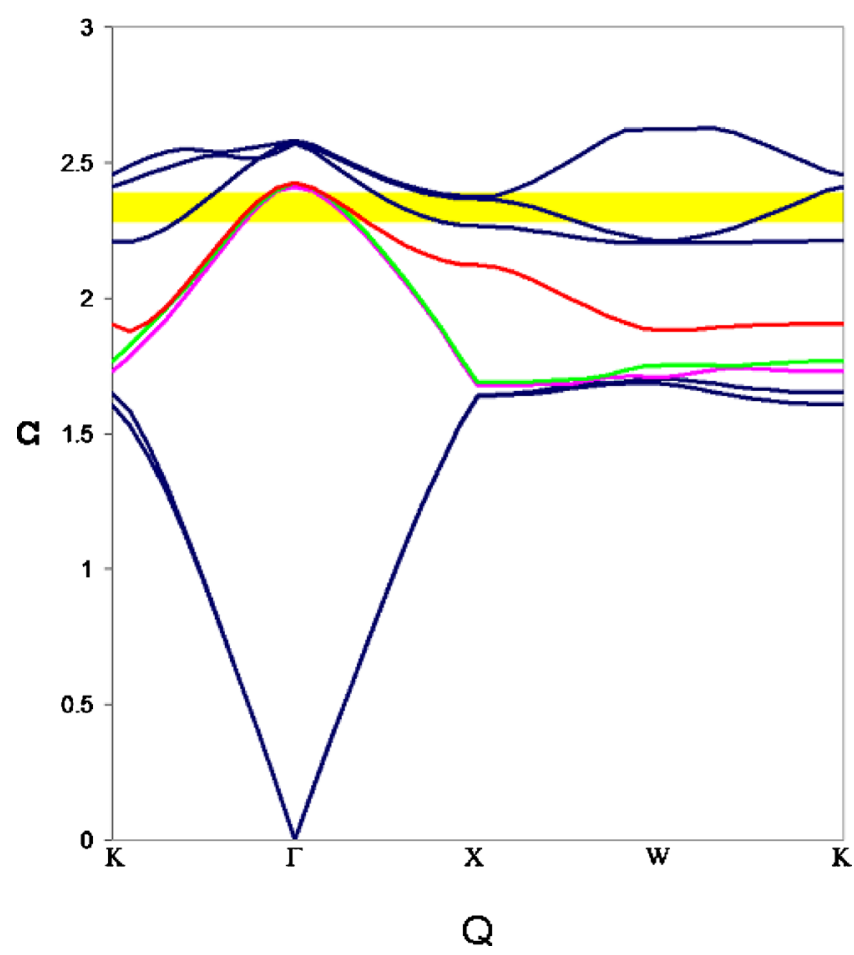

FIG. 4. (Color online) Polaritonic band structure of the fcc crystal of spherical cavities. Parameters: $\varepsilon_{\mathrm{s}}=1, r=0.32 a$, and $\varepsilon_{\mathrm{b}}=30$. The dimensionless wave vector and frequency are $Q=k a$ and $\Omega=\omega a / c$, respectively.

does depend strongly on the slab's edges and orientation. The configuration displayed here, in which the "cuts" were made along the $\Gamma-\mathrm{X}$ direction and directly through the sphere centers, was found to be optimal for this particular choice of parameters. In addition, inspection of the field distributions within the slab reveals the development of dipole moments in all directions, with a dominant response along the z-axis. The charge distribution depicted in Fig. 1 is clearly visible in the inset of Fig. 3, resulting from the scattering-induced excitation of electric fields in the $x-y$ plane.

\section{B. Lattice of spherical cavities}

The second crystal was chosen to be an fcc lattice of spherical cavities $\left(\varepsilon_{\mathrm{s}}=1\right)$ with radius $r=0.32 a$, embedded in a medium with $\varepsilon_{\mathrm{b}}=30$. The corresponding polaritonic band diagram is shown in Fig. 4. The most "spherical" EFS can be obtained at a frequency of $\Omega=2.26$. Even though multiple modes exist at this frequency, as long as the bands have very similar slopes and positions, an approximate, effective refractive index can still be meaningfully defined for the material at a single frequency, regardless of which modes are excited. The lensing through this crystal is displayed in Fig. 5. Interestingly, this system is less sensitive to slab termination, and a recognizable image exists for all terminations along the $\Gamma-\mathrm{X}$ axis. This is most likely due to the presence of multiple polaritonic bands at the frequency of interest, which increases the phase space for the photon-polariton scattering. 
(a)

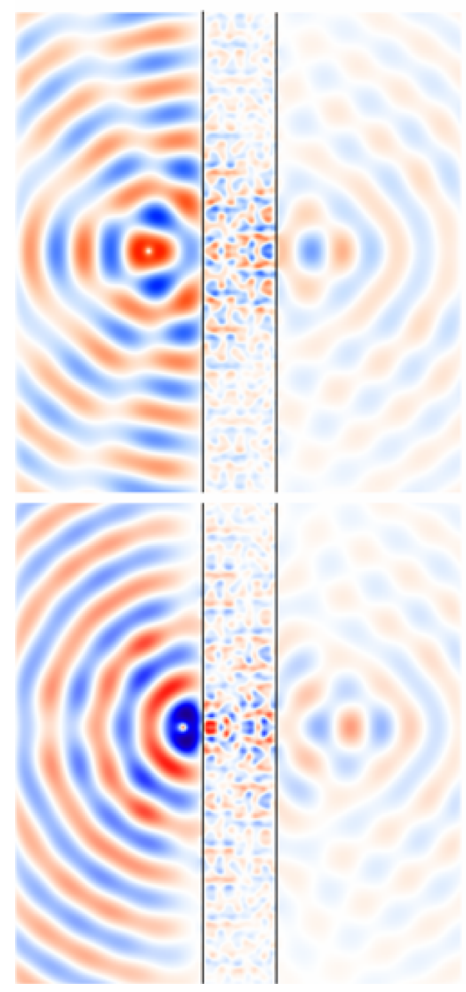

FIG. 5. (Color online) Map of the z-component of the electric field of a wave propagating through the fcc crystal slab of spherical cavities (parameters as in Fig. 4), at the frequency $\Omega=\omega a / c=2.26$, for two different locations of the source with respect to the slab. Colored figure available online. (b) Schematic of the light rays through a slab of a material with $n=-1$.

\section{FABRICATION PROPOSALS}

\section{A. Microwave regime}

There should be no difficulty in realizing these crystals for experiments in the microwave regime, given that there exist commercially available ceramic and polymeric composites with $\varepsilon_{\mathrm{s}}=30$ and negligible losses at this frequency range. We propose fabrication of the first fcc crystal by suspending ceramic spheres of radius $0.31 \mathrm{~cm}$ with a simple nylon thread network such that $a=1 \mathrm{~cm}$. For this setup, lensing should occur at a frequency of $10.2 \mathrm{GHz}$. The second crystal could be constructed from an fcc array $(a=1 \mathrm{~cm})$ of spherical air cavities (or other low dielectric constant material), each with a radius of $0.32 \mathrm{~cm}$, distributed in a ceramic/ polymeric dielectric matrix $\left(\varepsilon_{\mathrm{s}}=30\right)$. The lensing would be expected at a frequency of $10.8 \mathrm{GHz}$. These numbers, of course, are all scalable, depending on the available materials and equipment.

\section{B. Optical regime}

The far more challenging regime is the optical frequency range. This is due to the existence of fewer low loss materials having a sufficiently large refractive index at these frequencies. However, we observe that the proposed NR persists in materials with relatively large losses with little reduction in image quality. Returning to the first of our proposed systems, we found that a crystal with $a=266 \mathrm{~nm}$,

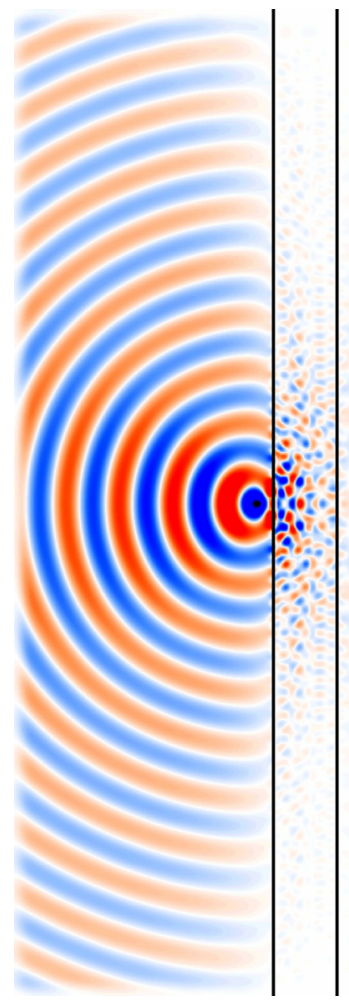

FIG. 6. (Color online) Map of the z-component of the electric field of a wave propagating through crystal slab of spheres (600 nm wavelength). Colored figure available online.

made of spherical nanoparticles of radius $74 \mathrm{~nm}(r=0.28 a)$ with $\varepsilon_{\mathrm{s}} \approx 20+0.2 i$ (corresponding to a wavelength of 600 $\mathrm{nm}$ ), still supports NR (see Fig. 6). As stated earlier, NR in a dipolar polaritonic crystal strongly relies on the intraparticle Mie resonances, ${ }^{25}$ which show high correlation to the NR condition in our crystals. This requirement of strong Mie resonances is precisely what prohibits the use of spheres with smaller dielectric constants. While there seems to be no single substance having the chosen value of $\varepsilon_{\mathrm{s}}$ at a wavelength of $600 \mathrm{~nm}$ (crystalline silicon is close with $\varepsilon_{\mathrm{s}} \approx 15.9$ +0.1 ), composite nanoparticles with enhanced Mie resonances can be used instead. It has recently been shown ${ }^{30}$ that the Mie resonances of metallic spherical shells can be much stronger (and narrower) than those of the corresponding metallic spheres of the same size, and that their resonance frequencies can be tuned (by adjusting the shell thickness) all across the visible range. It can be shown that the same is true for nonmetallic nanoparticles of sufficiently large dielectric constant. $^{31}$ Thus, the requirement of sufficiently strong Mie resonance is actually much less restrictive than the requirement of sufficiently high dielectric constant. This, in turn, allows for a variety of core-shell nanoparticle combinations that can give effectively the same contribution as the pure dielectric spheres used in our simulations, provided that the loss in these nanoparticles remains within the acceptable limit. This wide berth in composition and frequency of operation gives our proposed crystal a level of configurability not usually found in optical metamaterials. In addition, the tuning of the first and second Mie resonances for a given lattice constant can lead to better fulfillment of Eq. (5), which was shown earlier to be a rough quality factor for our 
system. Thus, our proposal represents a polaritonic avenue toward optical metamaterials, and is achievable through available materials and fabrication techniques.

\section{CONCLUSION}

In conclusion, we have proposed two practical, complementary realizations of a point-dipole crystal, shown earlier to be capable of NR on a polaritonic band: an array of highly polarizable spheres, and the complementary array of spherical cavities in a highly polarizable medium. We show that, for the right choice of parameters, these crystals are capable of NR. We also propose how to demonstrate this NR in both the microwave and optical regimes using available materials. In the latter case, we propose to exploit the intraparticle nature of polaritonic crystals, employing composite nanoparticles with enhanced Mie resonances to achieve NR. An experimental attempt to complement these results is strongly encouraged.

\section{ACKNOWLEDGMENTS} Inc.

This work was supported in part by a grant from Solasta,

${ }^{1}$ V. G. Veselago, Sov. Phys. Usp. 10, 509 (1968).

${ }^{2}$ H. Kosaka, T. Kawashima, A. Tomita, M. Notomi, T. Tamamura, T. Sato, and S. Kawakami, Phys. Rev. B 58, R10096 (1998).

${ }^{3}$ J. B. Pendry, A. J. Holden, W. J. Stewart, and I. Youngs, Phys. Rev. Lett. 76, 4773 (1996).

${ }^{4}$ J. B. Pendry, A. J. Holden, D. J. Robbins, and W. J. Stewart, IEEE Trans. Microwave Theory Tech. 47, 2075 (1999).

${ }^{5}$ D. R. Smith, W. J. Padilla, D. C. Vier, S. C. Nemat-Nasser, and S. Schultz, Phys. Rev. Lett. 84, 4184 (2000).

${ }^{6}$ C. L. Holloway, E. F. Kuester, J. Baker-Jarvis, and P. Kabos, IEEE Trans. Antennas Propag. 51, 2596 (2003).
${ }^{7}$ V. Yannopapas and A. Moroz, J. Phys.: Condens. Matter 17, 3717 (2005). ${ }^{8}$ M. S. Wheeler, J. S. Aitchison, and M. Mojahedi, Phys. Rev. B 72, 193103 (2005).

${ }^{9}$ L. Jylhä, I. Kolmakov, S. Maslovski, and S. Tretyakov, J. Appl. Phys. 99, 043102 (2006).

${ }^{10}$ D. R. Smith and N. Kroll, Phys. Rev. Lett. 85, 2933 (2000).

${ }^{11}$ R. A. Shelby, D. R. Smith, S. C. Nemat-Nasser, and S. Schultz, Appl. Phys. Lett. 78, 489 (2001).

${ }^{12}$ R. A. Shelby, D. R. Smith, and S. Schultz, Science 292, 77 (2001).

${ }^{13}$ R. W. Ziolkowski and E. Heyman, Phys. Rev. E 64, 056625 (2001).

${ }^{14}$ M. Bayindir, K. Aydin, E. Ozbay, P. Markos, and C. M. Soukoulis, Appl. Phys. Lett. 81, 120 (2002).

${ }^{15}$ R. M. Walser, A. P. Valanju, and P. M. Valanju, Phys. Rev. Lett. 87, 119701 (2001).

${ }^{16}$ D. R. Smith, D. Schurig, and J. B. Pendry, Appl. Phys. Lett. 81, 2713 (2002).

${ }^{17}$ J. B. Pendry, Phys. Rev. Lett. 85, 3966 (2000).

${ }^{18}$ P. V. Parimi, W. T. Lu, P. Vodo, J. Sokoloff, J. S. Derov, and S. Sridhar, Phys. Rev. Lett. 92, 127401 (2004).

${ }^{19}$ C. Luo, S. G. Johnson, J. D. Joannopoulos, and J. B. Pendry, Phys. Rev. B 65, 201104 (2002); C. Luo, S. G. Johnson, and J. D. Joannopoulos, Appl. Phys. Lett. 81, 2352 (2002).

${ }^{20}$ X. Wang, Z. F. Ren, and K. Kempa, Opt. Express 12, 2919 (2004).

${ }^{21}$ J. D. Joannopoulos, R. D. Meade, and J. N. Winn, Photonic Crystals: Molding the Flow of Light (Princeton University Press, Princeton, 1995).

${ }^{22}$ X. Wang and K. Kempa, Phys. Rev. B 71, 233101 (2005).

${ }^{23}$ K. Kempa and A. Rose, Bull. Pol. Acad. Sci.: Tech. Sci. 57(1), 35 (2009).

${ }^{24}$ A. Ahmadi and H. Mosallaei, Phys. Rev. B 77, 045104 (2008).

${ }^{25}$ K. Kempa, R. Ruppin, and J. B. Pendry, Phys. Rev. B 72, 205103 (2005).

${ }^{26}$ E. A. Yablonskii, N. A. Muljarov, N. A. Gippius, S. G. Tikhodeev, and T. Ishihara, Phys. Status Solidi A 190, 413 (2002).

${ }^{27}$ K. C. Huang, P. Bienstman, J. D. Joannopoulos, K. A. Nelson, and S. Fan, Phys. Rev. Lett. 90, 196402 (2003).

${ }^{28} \mathrm{~A}$. Taflove, Computational Electrodynamics-The Finite-Difference TimeDomain Method (Artech House, Norwood, MA, 1995).

${ }^{29}$ C. Vandenbem and J. P. Vigneron, J. Opt. Soc. Am. A 22, 1042 (2005).

${ }^{30}$ M. G. Blaber, M. D. Arnold, and M. J. Ford, J. Phys. Chem. C 113, 3041 (2009).

${ }^{31}$ M. Scharte, R. Porath, T. Ohms, M. Aeschlimann, J. F. Krenn, H. Ditlbacher, F. R. Aussenegg, and A. Liebsch, Appl. Phys. B: Lasers Opt. 73, 305 (2001). 